Ancient T-DNA integration events have been found in plant genomes ${ }^{5,6}$, and expression of these T-DNA sequences may have contributed to plant evolution ${ }^{7,8}$. Similarly, the presence of Agrobacterium chromosomal DNA in plants suggests intriguing evolutionary possibilities and expands our knowledge of horizontal gene flow between species.

Whereas plasmid backbone transfer was somewhat predictable from studies on T-DNA processing $^{9}$, the mechanism by which chromosomal DNA transfers to plants remains uncertain. The presence of insertion sequence (IS) elements in the AchrDNA in numerous T-DNA insertions in several insertion libraries led the authors to hypothesize that singlestranded T-DNA (the T-strand) processed from the T-DNA region may associate with nicks or breaks in bacterial chromosomal DNA resulting from IS element transposition. T-DNA integrated into these breaks would be 'relaunched' after reconstituting a new right border sequence (Fig. 1a). Such a border reconstruction could occur because sequences that serve as T-DNA borders can be somewhat degenerate ${ }^{10}$.

Other mechanisms for T-DNA linkage to bacterial chromosomal DNA are possible.For example, the T-strand could directly ligate to DNA at nick sites and transfer chromosomal DNA (Fig. 1b), or T-DNA could integrate into bacterial chromosomal DNA by homologous recombination, generating strains that behave like the Hfr (Fig. 1c) or $\mathrm{F}^{\prime}$ strains of Escherichia coli (Fig. 1d).

Although the authors favor the T-DNA chromosomal integration and border reconstitution model, there are no characterized Agrobacterium enzymatic activities that would allow T-strand integration into the bacterial chromosome or direct T-strand linkage to nicks in bacterial chromosomal DNA. Moreover, it is likely that the VirD2/T-strand complex is exported to the plant as soon as it is generated, preventing access to and recombination with the chromosome. Creation of Hfr or F'-like intermediates would require homology between DNA sequences within T-DNA and sequences in the bacterial chromosome. Although duplicated sequences such as IS elements may exist among natural T-DNA regions and the chromosome, they are unlikely to exist in the numerous different 'engineered' T-DNAs used to generate the disparate T-DNA insertion libraries.

Several other mechanisms may enable linkage of T-DNA to bacterial DNA. T-strands from the T-DNA region linked to T-strands generated by nicking of border-like sequences in the chromosome may integrate into the same site in plant DNA (Fig. 1e). Linkage of two different T-DNAs has been described previously ${ }^{11}$.
Alternatively, IS elements within the bacterial chromosome may link bacterial DNA with T-region DNA during a process similar to that of replicative transposition (Fig. 1f). These two latter models do not require homology between the T-region and the bacterial chromosome.

Whatever the mechanism, the introduction of bacterial chromosomal DNA into transgenic plants is not welcome news for regulators. The transfer of plasmid vector backbone sequences has raised the possibility that antibiotic resistance genes on the plasmid might be transferred to plants. T-DNA-encoded genes are transcribed from typical eukaryotic promoters. However, transcription in plants of bacterial chromosomal genes from prokaryotic promoters would be problematic.

The likelihood that approved transgenic crops might carry Agrobacterium chromosomal genes seems negligible. Genetically modified crops are routinely analyzed to determine the insertion site of T-DNA into plant DNA, and the presence of plasmid backbone or chromosomal DNA linked to T-DNA would be detected in these studies before field release. Moreover, as transgenic plants are routinely backcrossed many times to segregate out random mutations generated during T-DNA transfer and integration, any bacterial chromosomal DNA unlinked to the selected T-DNA would likely be eliminated during this stage of the breeding process.

High-throughput sequencing technologies should allow the rapid identification of bacterial chromosomal DNA integrated into the plant genome and the elimination of such transgenic plants from breeding programs. In addition, mutant Agrobacterium strains that would mitigate T-region recombination with the bacterial chromosome could be used to generate transgenic plants, thereby reducing the probability of bacterial chromosomal DNA transfer to plants.

1. Kononov, M.E., Bassuner, B. \& Gelvin, S.B. Plant J. 11 945-957 (1997).

2. Ülker, B. et al. Nat. Biotechnol. 26, 1015-1017 (2008).

3. Wood, D.W. et al. Science 294, 2317-2323 (2001).

4. Goodner, B. et al. Science 294, 2323-2328 (2001).

5. Furner, I.J. et al. Nature 319, 422-427 (1986).

6. Suzuki, K., Yamashita, I. \& Tanaka, N. Plant J. 32, 775-787 (2002).

7. Meyer, A.D., Ichikawa, T. \& Meins, F. Mol. Gen. Genet. 249, 265-273 (1995)

8. Ichikawa, T., Ozeki, Y. \& Syono, K. Mol. Gen. Genet. 220, 177-180 (1990).

9. Durrenberger, F., Crameri, A., Hohn, B. \& KoukolikovaNicola, Z. Proc. Natl. Acad. Sci. USA 86, 9154-9158 (1989).

10. Rommens, C.M. et al. Plant Physiol. 139, 1338-1349 (2005).

11. De Neve, M. et al. Plant J. 11, 15-29 (1997).

\title{
Viral attenuation by design
}

\author{
Harriet L Robinson
}

\section{A less-virulent poliovirus generated by altering codon usage suggests a new strategy for vaccine development.}

Although live attenuated viruses provide the most effective vaccination strategy ever devised, current research has largely abandoned classical methods for vaccine development in favor of 'high-tech' approaches such as protein subunit and recombinant DNA vaccines. A recent study ${ }^{1}$ in Science describes a method for reducing viral virulence that may eventually lead to a new class of live attenuated vaccines. Manipulation of codon usage has been exploited previously to increase the expression of select antigens in DNA vaccines ${ }^{2}$; Coleman et al. ${ }^{1}$ apply it to opposite effect-to decrease gene expression in poliovirus by introducing underrepresented codon pairs (Fig. 1).

\section{Harriet L. Robinson is at GeoVax, Inc., 1256 Briarcliff Road NE, Atlanta, Georgia 30306,} USA.

e-mail: hrobinson@geovax.com
Live attenuated vaccines have saved millions of lives and days of sickness by eliciting long-lasting antibody and T-cell responses ${ }^{3}$. Examples include the highly successful smallpox, measles, mumps, rubella, chicken pox and oral polio vaccines. Unlike inactivated, subunit or recombinant protein vaccines (such as those against diphtheria, pertussis, tetanus, hepatitis B virus and flu), which require multiple doses and regular boosting, live attenuated vaccines provided as single doses elicit long-lasting protection. Moreover, they are the most cost-effective form of vaccination. Manufacture requires only the growth of the virus. Purification is minimal because the vaccines, which amplify as they vaccinate, are effective at low doses. Live attenuated vaccines have the additional benefit of not requiring adjuvants: replicating pathogens provide the reservoirs of antigen and 'danger signals' needed to mobilize the immune response that 
adjuvants provide for nonreplicating vaccines.

Despite the tremendous advantages of live attenuated vaccines, current vaccine research is centered on the use of crystal structures to design protein subunit vaccines ${ }^{4}$, of databases to develop inserts for recombinant viral and DNA vaccines ${ }^{5}$ and of cell-signaling microarrays to choose adjuvants ${ }^{6}$. Gone are the days when live attenuated vaccines were developed with biological systems, such as serial passage of a pathogen (mumps and measles vaccines), testing of virus derived from individual virions (poliovirus vaccine) or the use of related viruses from other species (smallpox vaccine). Coleman et al. ${ }^{1}$ have provided a contemporary approach for the development of live attenuated vaccines by showing that a pathogen's virulence can be restricted simply by engineering its codon pair bias. Their approach, termed synthetic attenuated virus engineering (SAVE), involves substitution of synonymous codons to reduce the codon pair bias characteristic of the wild-type virus.

Specifically, the authors used recombinant DNA technology to produce a poliovirus in which the 5 -most coding sequence, for the capsid protein $\mathrm{P} 1$, was recoded with 631 synonymous mutations to give a codon pair bias $\sim 4.5$ fold lower than that of wild-type poliovirus. The attenuated virus was further manipulated by recombining segments of wild-type polio sequences into the codon pair-biased sequence to generate two viable viruses with reduced translation and replication.

How well did manipulation of codon pair bias translate to vaccine efficacy in vivo? Compared with wild-type virus, the two attenuated viruses required tenfold higher doses of plaque-forming units to kill transgenic mice expressing the poliovirus receptor. In a pilot vaccination study, three high doses of the attenuated vaccines given at weekly intervals resulted in the death of 9 of 16 mice but protected the 7 survivors against a paralytic polio challenge. The codon pair bias selection resulted in two attenuated viruses that are only about tenfold attenuated in their 50\% paralytic dose and, at the same time, at least tenfold attenuated in their ability to be manufactured ${ }^{1}$. In contrast, the selection of individual infectious units of poliovirus for attenuated neurotropism resulted in an oral vaccine that is $>500,000$-fold attenuated in its 50\% paralytic dose without any attenuation of its ability to be produced ${ }^{7}$. Thus, selection against neurotropism to create the current polio vaccine, which involves three key codons ${ }^{7}$, yielded a
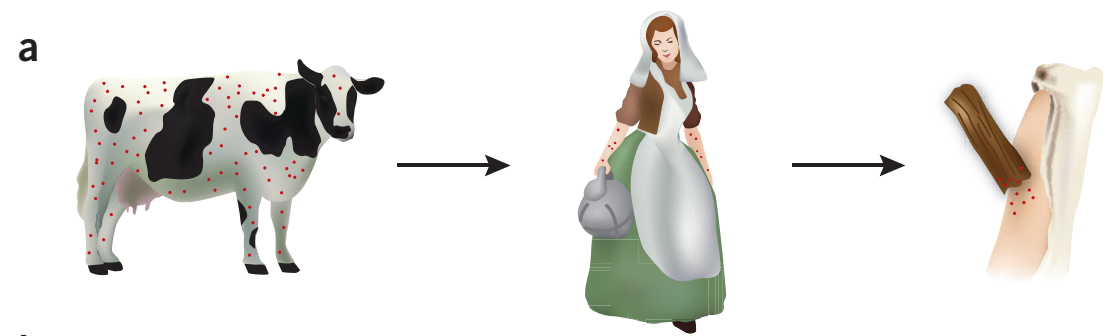

b

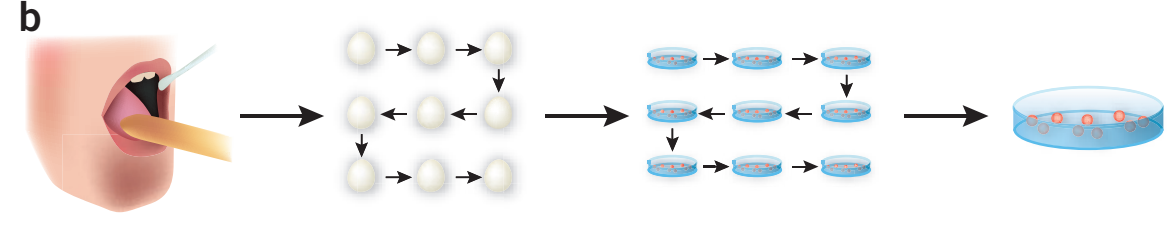

C
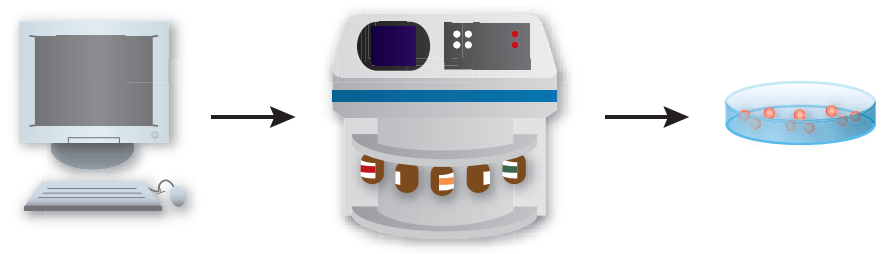

Figure 1 Development of an attenuated vaccine by two classical methods and the proposed method of Coleman et al. ${ }^{1}$. (a) A virus from one species is used in another species. Edward Jenner used fluid from a milk maid's acquired case of cowpox to demonstrate the protective potential of the cowpox virus for human smallpox ${ }^{8}$. (b) Serial passage in chicken cells. Maurice Hilleman recovered mumps virus from a clinical case in his 6-year-old daughter and passed the recovered virus in embryonated eggs and then in cultured chicken cells to produce the Jeryl Lynn mumps vaccine ${ }^{9}$. (c) Manipulation of codon pair bias. Coleman et al. ${ }^{1}$ used a computational algorithm to design a sequence with codon pair bias, an automated DNA synthesizer to construct the generated sequence, and transfected cultures to recover an attenuated poliovirus.

much more effective vaccine than the 631 mutations in capsid protein P1 (ref. 1).

Efforts to eradicate polio have suffered more from a lack of political will and popular fears than from limitations on the ability to manufacture and deliver the current oral vaccines. Although the use of SAVE to produce growthattenuated polioviruses is unlikely to yield a vaccine that is superior to existing polio vaccines, this strategy may prove valuable in the case of other infectious diseases for which there are no effective vaccines. However, any candidate attenuated virus generated by this approach would require attenuation for disease prevention and not only for growth. For example, our current highly successful polio vaccines were selected for the following: "(1) maintaining high degree of infectivity in cell culture and the human intestinal tract, (2) inducing detectable levels of neutralizing antibody in a high proportion of susceptible (seronegative) recipients, (3) displaying low neurovirulence in monkeys,
(4) demonstrating a lack of association with paralytic disease in humans, and (5) maintaining genetic stability after replication in the human host" ${ }^{\prime 7}$. Thus, the study of Coleman et al. ${ }^{1}$ opens a new path to attenuation that despite its universality will have to be tailored to the pathogenesis of each agent to be useful for future vaccine development.

1. Coleman, J.R. et al. Science 320, 1784-1787 (2008).

2. Deml, L. et al. J. Virol. 75, 10991-1001 (2001).

3. Nathanson, N. \& Robinson, H.L. in Viral Pathogenesis and Immunity (ed. Nathanson, N.) 234-252 (Academic Press, Amsterdam, 2007).

4. Burton, D.R. et al. Nat. Immunol. 5, 233-236 (2004).

5. Korber, B., LaBute, M. \& Yusim, K. PLoS Comput. Biol. 2, e71 (2006).

6. Bendelac, A. \& Medzhitov, R. J. Exp. Med. 195, F19F23 (2002).

7. Sutter, R.W., Cochi, S.L. \& Melnick, J.L. in Vaccines (eds. Plotkin, S.A. \& Orenstein, W.A.) 364-408 (W.B. Saunders, Philadelphia, 1999).

8. Jenner, E. An Inquiry into the Causes and Effects of the Variolae Vaccinae (Low, London, 1798).

9. Buynak, E.B. \& Hilleman, M.R. Proc. Soc. Exp. Biol. Med. 123, 768-775 (1996). 